\title{
Image, événement, durée
}

\section{Georges Didi-Huberman}

\section{OpenEdition}

\section{Journals}

Édition électronique

URL : http://journals.openedition.org/imagesrevues/787

DOI : 10.4000/imagesrevues.787

ISSN : 1778-3801

\section{Éditeur :}

Centre d'Histoire et Théorie des Arts, Groupe d'Anthropologie Historique de l'Occident Médiéval, Laboratoire d'Anthropologie Sociale, UMR 8210 Anthropologie et Histoire des Mondes Antiques

\section{Référence électronique}

Georges Didi-Huberman, «Image, événement, durée », Images Re-vues [En ligne], Hors-série 1 | 2008, document 1, mis en ligne le 21 avril 2011, consulté le 30 janvier 2021. URL : http://

journals.openedition.org/imagesrevues/787 ; DOI : https://doi.org/10.4000/imagesrevues.787

Ce document a été généré automatiquement le 30 janvier 2021.

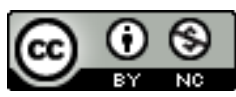

Images Re-vues est mise à disposition selon les termes de la Licence Creative Commons Attribution -

Pas d'Utilisation Commerciale 4.0 International. 


\title{
Image, événement, durée
}

\author{
Georges Didi-Huberman
}

1 La durée se construit à chaque moment dans un certain rapport entre histoire et mémoire, présent et désir. Mais cette construction se heurte toujours aux obstacles de l'habitude. Par exemple: nous avons, comme tous les jours, regardé les informations télévisées. Nous avons revu, pour la énième fois, ces images de feu et de sang, de guerres et de souffrances humaines. Mais sont-elles

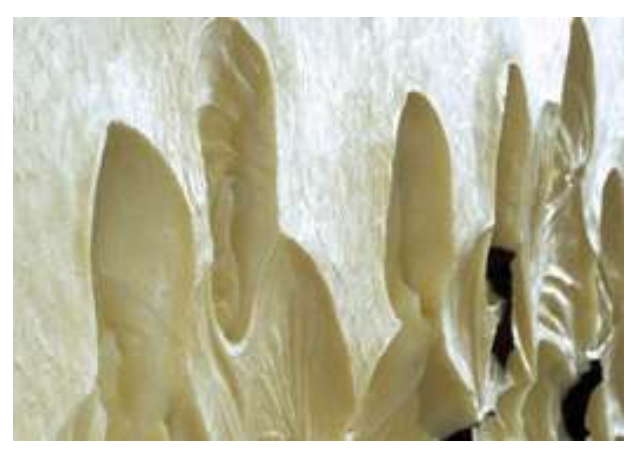
seulement des images? Ne sont-elles pas plutôt de simples extraits, au sens où l'on parle d'un extrait de film, sortes de clips des malheurs historiques qu'elles sont censées documenter? Ne sont-elles pas, dès lors, impossibles à regarder vraiment, c'est-à-dire incapables de se construire en durée, montées qu'elles sont à la manière des spots publicitaires, écrasées dans un nivellement général - est-ce aveuglement ou bien cynisme ? On ne sait plus trop bien - avec le tout-venant de la société du spectacle, assourdies dans la langue de bois médiatique, noyées dans le flux qui les emporte, le flux des clichés où nous trouvons, à bon compte, nos simulacres de repères et de significations?

2 Une œuvre récente de Pascal Convert (fig. 1-4), élément d'un travail plus extensif sur quelques images de malheurs historiques ${ }^{1}$, propose une expérience bien différente. 
Fig. 1

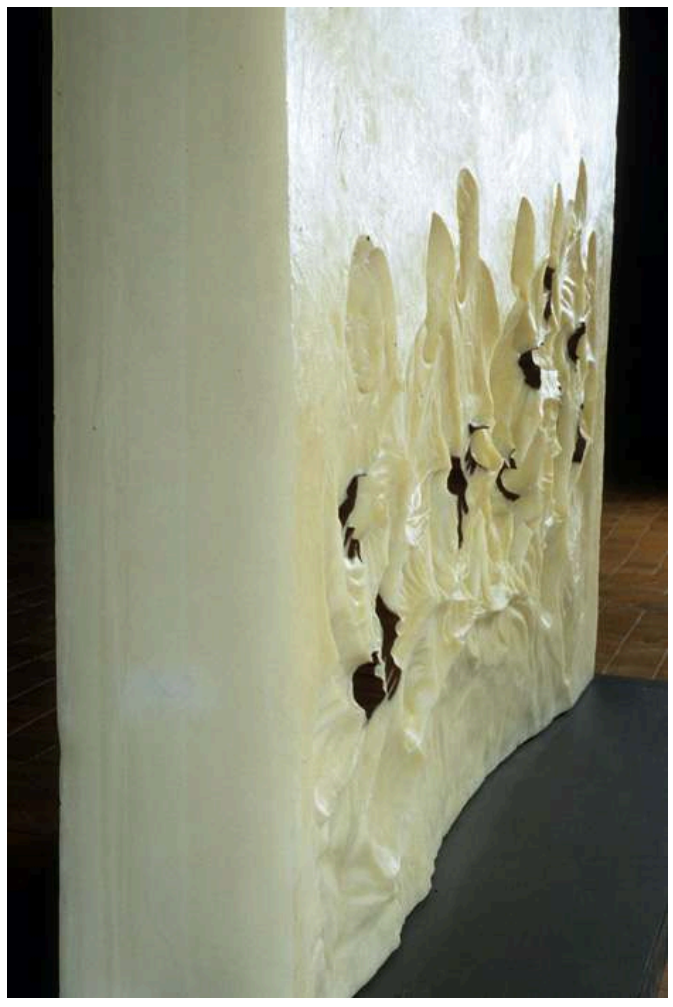

Pascal Convert, Sans titre (inspiréede Veillée funèbre au Kosovo, photographie de Georges Mérillon, 1990). Cire, résine et cuivre, $224 \times 278 \times 40 \mathrm{~cm}$.

Luxembourg, Musée d'Art moderne Grand-Duc Jean

3 Nous ne sommes plus en face d'un écran où l'on pourrait zapper dès que surgit l'ennui ou le début de l'angoisse; mais devant une sorte de pan. Masse et obstacle: un mur blanc, inévitable. Légèrement concave (fig. 1-2), traversé par d'étranges remous de surface, il nous attire vers sa courbure, nous appelle physiquement, nous invite à l'approche; mais son opacité soudain nous surplombe, nous barre la route. Façon de nous dérouter en arrêtant notre pas, façon de nous désorienter en nous immobilisant devant lui. 
Fig. 2

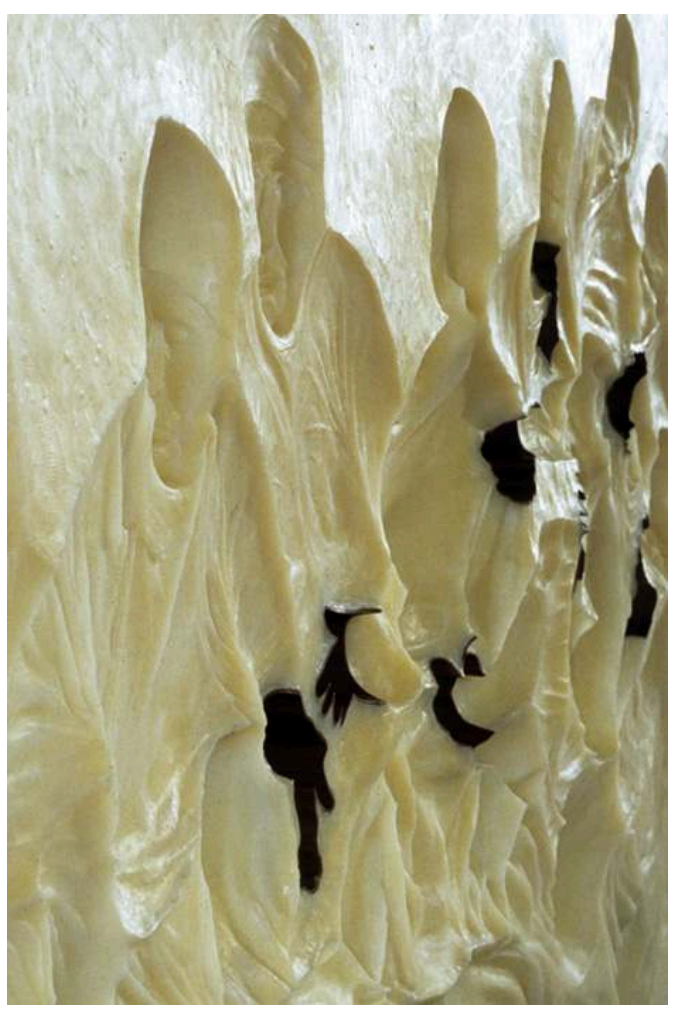

Pascal Convert, Sans titre (inspiréede Veillée funèbre au Kosovo, photographie de Georges Mérillon, 1990). Cire, résine et cuivre, $224 \times 278 \times 40 \mathrm{~cm}$.

Luxembourg, Musée d'Art moderne Grand-Duc Jean

Ce mur est fait d'une surprenante matière : les mouvements qui l'agitent font d'abord penser aux reliefs de Donatello, mais l'objet n'a pas du tout la consistance du marbre. Cela évoquerait plutôt l'albâtre, car la lumière pénètre jusqu'à une certaine profondeur et laisse comme une - magnifique -aura dans la masse. On comprend soudain que ce mur est tout entier de cire blanche. On comprend, ensuite, que ses "reliefs » sont surtout faits de creux, de creusements blancs avec, ici et là, formant constellations, quelques trous sombres où s'indique une matière différente. L'expérience visuelle devient complexe : ici l'obstacle s'impose, là une trouée invite à traverser le mur ; ici la lumière irradie de l'intérieur, là elle s'accroche et, même, écorche les formes; ici elle accentue, là elle dissout ; ici la matérialité s'accuse, là se crée de l'intangible. On échoue d'emblée à saisir le jeu du positif et du négatif, des reliefs et des creux, la reproduction photographique ne faisant que pousser au comble ce phénomène d'ambiguïté (fig. 3). 
Fig.3

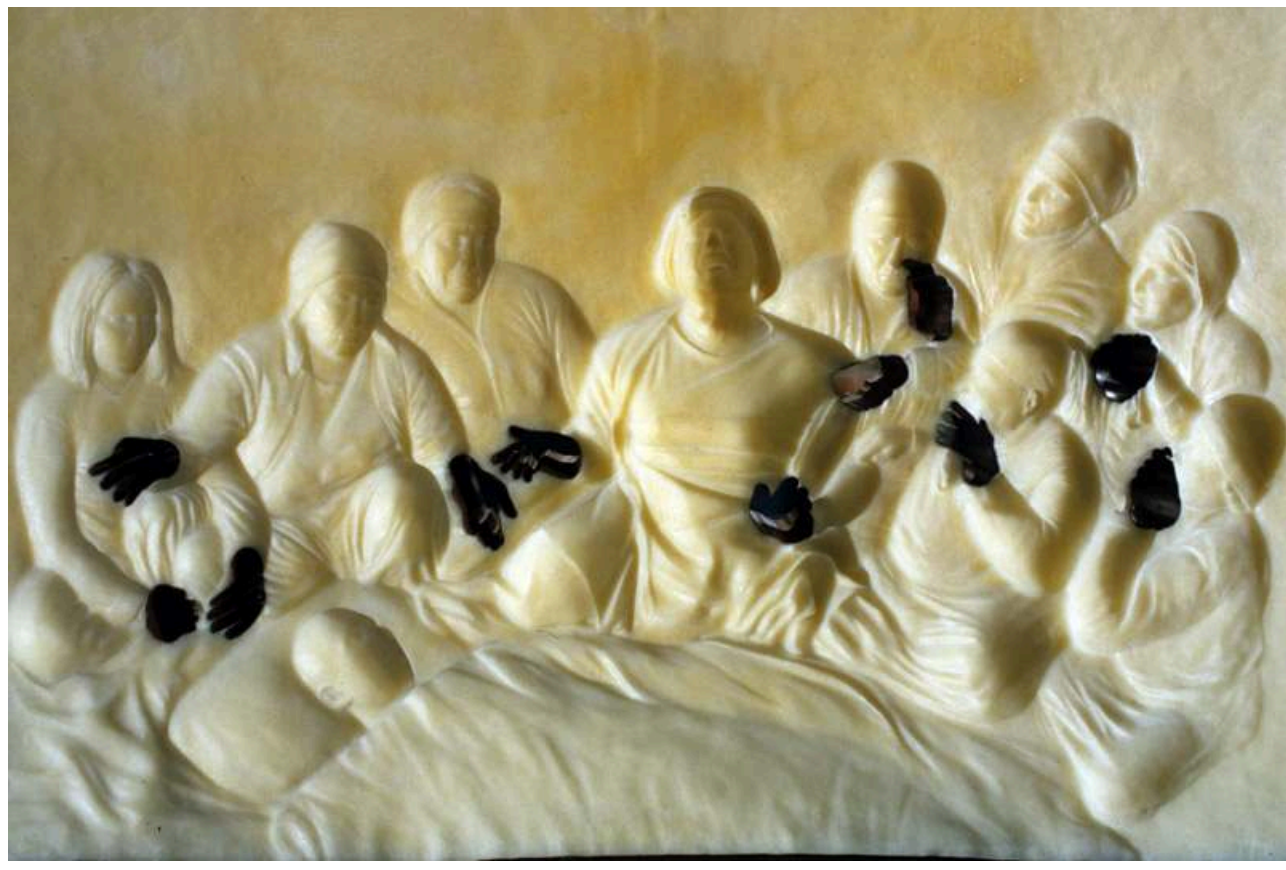

Pascal Convert, Sans titre (inspiréede Veillée funèbre au Kosovo, photographie de Georges Mérillon, 1990). Cire, résine et cuivre, $224 \times 278 \times 40 \mathrm{~cm}$.

Luxembourg, Musée d'Art moderne Grand-Duc Jean

5 Nous sommes bien loin de tout cliché : rien, dans cette masse, ne nous donne le signe disponible, l'extrait reconnaissable, le clip ou le spot de quoi que ce soit. Le langage luimême est privé de ses propres clichés. Devant cette masse de silence, les repères de signification ne sont pas donnés à l'avance : ils sont à construire sur le moment de mutité qui nous saisit d'abord, nous interloque. Il faut donc un peu de patience : on regarde, on attend, on questionne. Tout à coup, surgit ce qu'on n'attendait pas : et l'on découvre que ce frémissement blanc, agité comme une grande draperie, est, en réalité, une image à tue-tête, une image qui hurle, qui tempête, qui proteste devant nous. Toutes ces vagues de cire drapée, tous ces creux ne sont que les formes de cris et de douleurs émanés d'une dizaine de figures féminines réunies autour d'un corps étendu, probablement un cadavre.

6 La reconnaissance figurative est, certes, rendue difficile par l'omniprésence des formes négatives dans cette sculpture. Mais elle est immédiate pour une autre raison: toutes ces figures de femmes sont, devant nous, représentées à l'échelle humaine, leur visage ayant, plus ou moins, la dimension du nôtre. À cette différence près qu'il forme une matrice en creux où le nôtre pourrait presque s'inscrire ou se lover. Or, ce grand remous de béances accuse encore, sur la paroi blanche de la sculpture, l'expressivité de toute la scène : ce ne sont pas seulement neuf femmes qui se lamentent devant un corps mort - tout cela traité en négatif -, c'est comme un mur de lamentations que nous avons devant nous, un mur tout entier pétri de cris ouverts, un mur où tout devient « bouches débridées ", " déraison » et "malheur ", selon le vocabulaire tragique d'Euripide dans Les Bacchantes ${ }^{2}$. Partout où s'agitent les mains de ces femmes - gestes de l'affliction et du deuil -, Pascal Convert a choisi de radicaliser le creux par un réseau d'orifices qui traversent la paroi, en sorte que le revers du mur ne présente plus au regard qu'une constellation de mains négatives (fig. 4). 
Fig.4

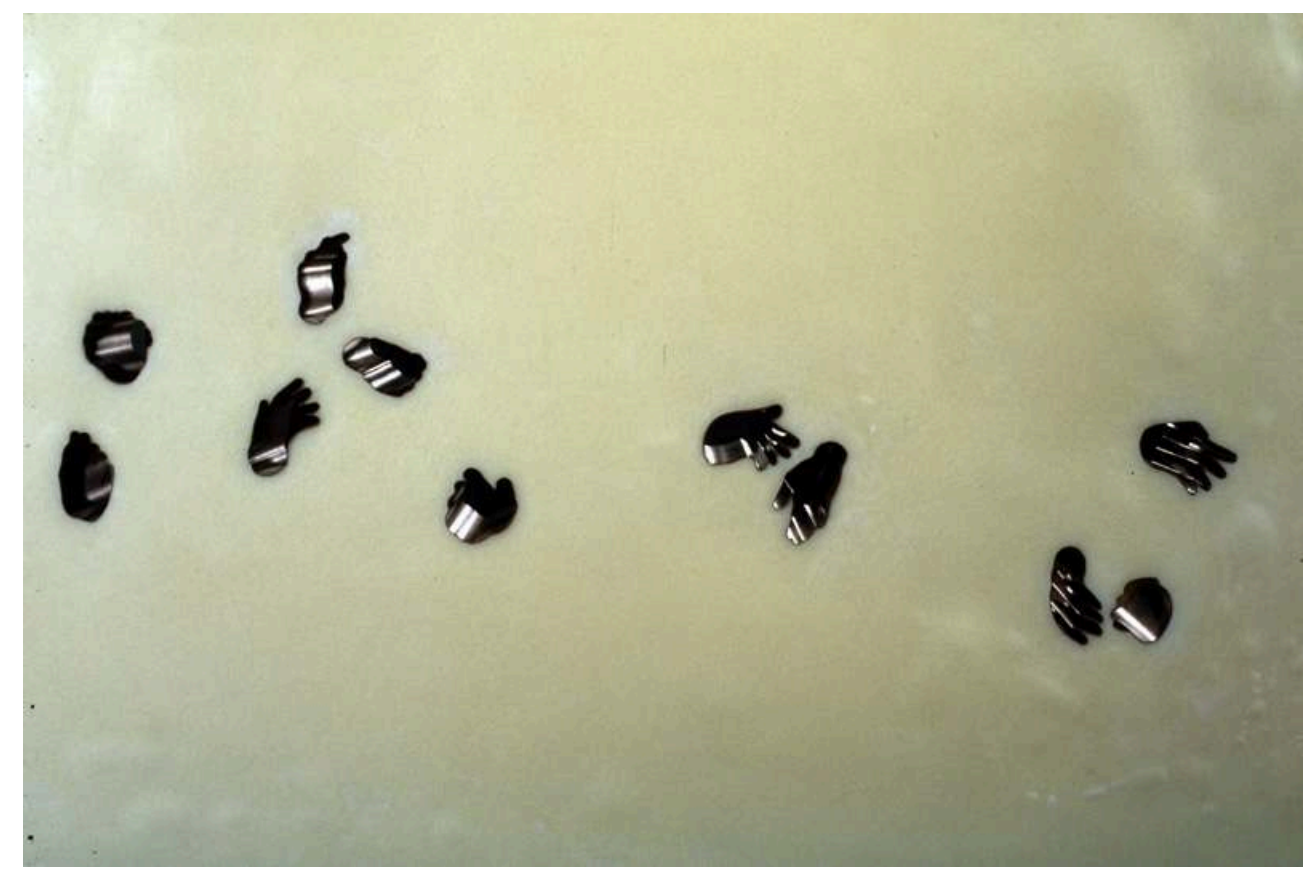

Pascal Convert, Sans titre (inspirée de Veillée funèbre au Kosovo, photographie de Georges Mérillon, 1990). Cire, résine et cuivre, $224 \times 278 \times 40 \mathrm{~cm}$.

Luxembourg, Musée d'Art moderne Grand-Duc Jean

7 Utiliser le creux pour sculpter le cri: Convert prend ici position dans un débat esthétique de très longue durée. Il ne renonce - comme voulait y obliger l'ascétisme de Clement Greenberg dans son New Laocoon ${ }^{3}-$ ni à la figure, ni au référent, ni à l'expression, ni même au pathos le plus extrême. Il ne renonce pas à sculpter les «bouches débridées" par la douleur, que Lessing avait, dans son propre Laocoon, proscrit de la façon la plus nette :

«Imaginez Laocoon la bouche béante et jugez. Faites-le crier et vous verrez. C'était une image qui inspirait la compassion parce qu'elle incarnait simultanément la beauté et la douleur ; maintenant c'est une image hideuse, monstrueuse, dont on voudrait détourner son regard parce que la vue de la douleur excite la répugnance sans que la beauté de l'objet souffrant puisse muer cette répugnance en un doux sentiment de compassion.

Une bouche béante est, en peinture, une tache, en sculpture un creux, qui produisent l'effet le plus choquant du monde, sans parler de l'aspect repoussant qu'elle donne au reste du visage tordu et grimaçant ${ }^{4}$. »

8 Cette interdiction était d'autant plus significative, dans l'idée de Lessing, qu'elle prétendait énoncer les limites intrinsèques de toute image visuelle face à la temporalité, à la durée de son référent : l'image ne représente qu'un " unique instant ", écrit Lessing; " Or, dans le cours d'une passion, l'instant du paroxysme est celui qui jouit le moins de ce privilège [qu'offre l'imagination du temps]. Au-delà, il n'y a plus rien, et présenter aux yeux le degré extrême, c'est lier les ailes à l'imagination »; les guerriers blessés qui tombent en criant chez Homère, les mères tragiques qui se lamentent, la façon générale qu'avaient les anciens Grecs d'« être braves sans cesser de pleurer ", tout cela est péremptoirement assigné par Lessing au seul pouvoir de l'écriture poétique ${ }^{5}$. Les sculptures, elles, n'ont qu'à voiler leurs cris. Ce précepte n'aura 
pas empêché toute une tradition sculpturale - depuis l'Antiquité alexandrine jusqu'à Donatello, depuis Guido Mazzoni et Niccolò dell'Arca jusqu'au Bernin, depuis Franz Xaver Messerschmidt jusqu'à Medardo Rosso - de "débrider les bouches». Et comment se défaire, devant notre mur blanc aux étranges surfaces agitées - plissées, creusées, trouées -, de l'impression qu'il réussit à présenter le cri avec sa propre draperie, la noire béance douloureuse avec sa propre pudeur, c'est-à-dire sa propre blancheur?

9 L'œuvre blême de Pascal Convert se présente, en réalité, comme l'interprétation d'une autre image - une image qui ne fut ni pensée, ni produite comme œuvre d'art, mais dont l'enjeu était d'informer sur un événement, de rendre visible un fait criant : c'est une image - en couleurs - prise par Georges Mérillon, photographe de l'agence Gamma, dans un village du Kosovo le 29 janvier 1990. Cela se passait donc presque dix ans avant la "Guerre du Kosovo» qui déchira la région entre mars 1998 (date des premières attaques massives des forces serbes dans la Drenica) et juin 1999 (date d'entrée de la KFOR au Kosovo depuis la Macédoine et l'Albanie, après que le Tribunal pénal international eut inculpé Slobodan Milosevic de crimes de guerre, et que l'OTAN eut mis en œuvre la résolution 1244 de l'ONU instaurant une présence internationale au Kosovo). Autant dire qu'à cette époque, nul ne pensait qu'il faille prêter attention aux troubles qui opposaient à l'armée serbe l'ardeur désarmée des jeunes nationalistes kosovars.

10 Georges Mérillon avait vu tomber les dépêches de l'AFP sur ces premières manifestations. Il était donc parti pour Pristina, sans information précise, sans traducteur. Il assista, dès le premier jour, à quelques heurts entre les jeunes autonomistes et les forces serbes : "On en était encore à jeter des cailloux contre des blindés ", expliquera-t-il à Pascal Convert ${ }^{6}$. Le soir du deuxième jour, il entendit parler d'un village où l'armée avait tiré à balles réelles sur les manifestants. Il s'y rendit le lendemain, avec le photographe serbe Dusko Despotovic, Milos Cvetkovic et une petite équipe de la télévision française dirigée par Véronique Taveau. Ils atteignirent le village de Nagafc en fin de matinée : impression de « bout du monde ${ }^{7}$ ».

11 Et Mérillon de raconter comment les villageois se précipitent vers les nouveaux arrivants pour dire leur douleur, leur colère. Le 27 janvier, la police serbe a littéralement tendu une embuscade aux quelques jeunes du village qui voulaient se rendre au rassemblement de Rahovec, à quelques kilomètres de là. Elle s'est postée dans un virage et elle a tiré : quatre morts, trente-deux blessés. Les journalistes sont presque emmenés de force dans une petite pièce où gît le cadavre d'un militant de vingt-huit ans, Nasimi Elshani. Les femmes - la mère du jeune homme, ses sœurs, son épouse, les amies - sont toutes là, serrées les unes contre les autres, autour du corps déposé à terre. Mais il y a urgence: le couvre-feu exige que l'on reparte au plus vite vers Pristina. L'équipe de Véronique Taveau - trois personnes en tout - filme le groupe des femmes (fig. 5). 
Fig.5

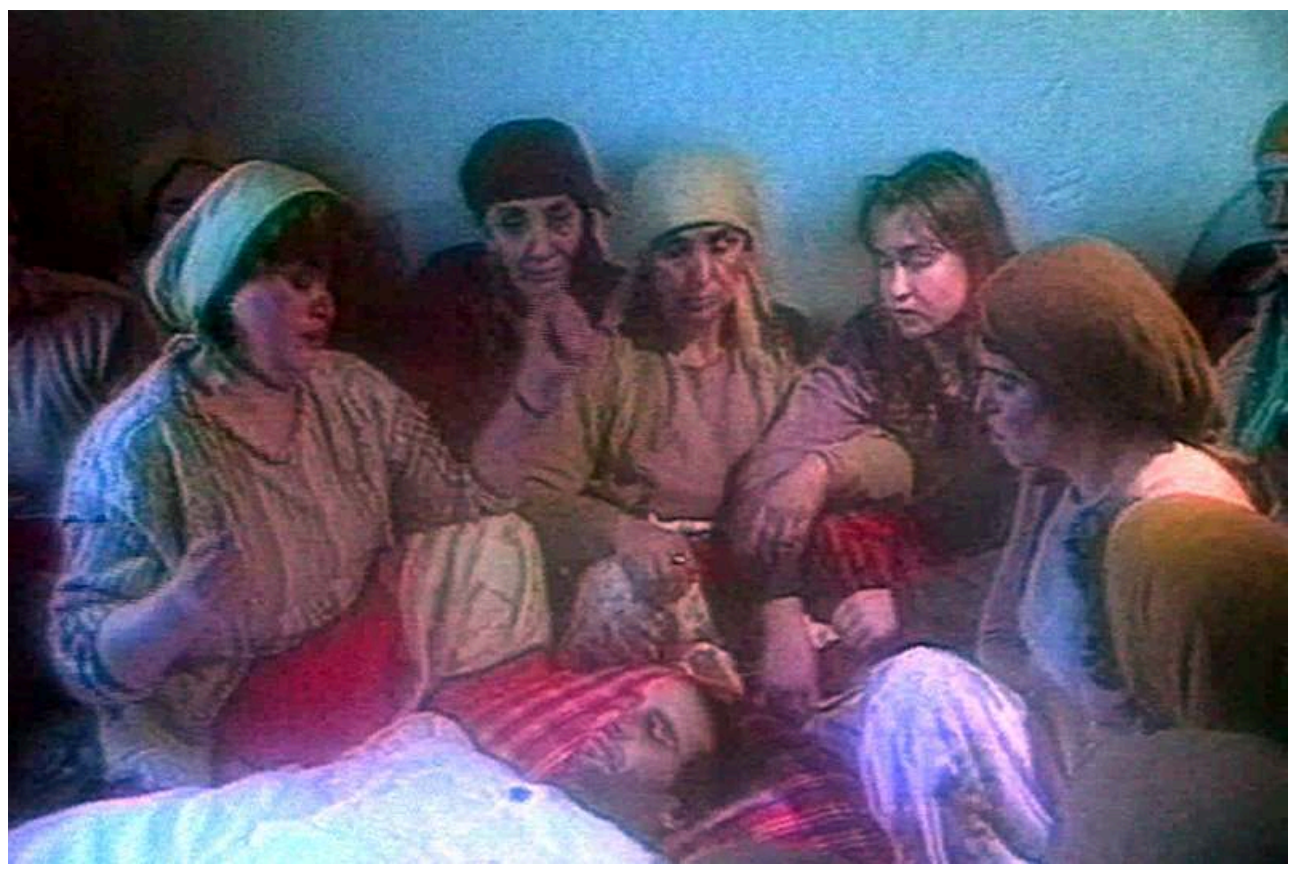

Véronique Taveau, Veillée funèbre au Kosovo, 1990

Vidéogramme tiré du journal télévisé d'Antenne-2 (31 janvier 1990)

12 Dusko Despotovic profite de la vive lumière fournie par le spot de la caméra pour réaliser quelques instantanés (fig. 6).

Fig.6

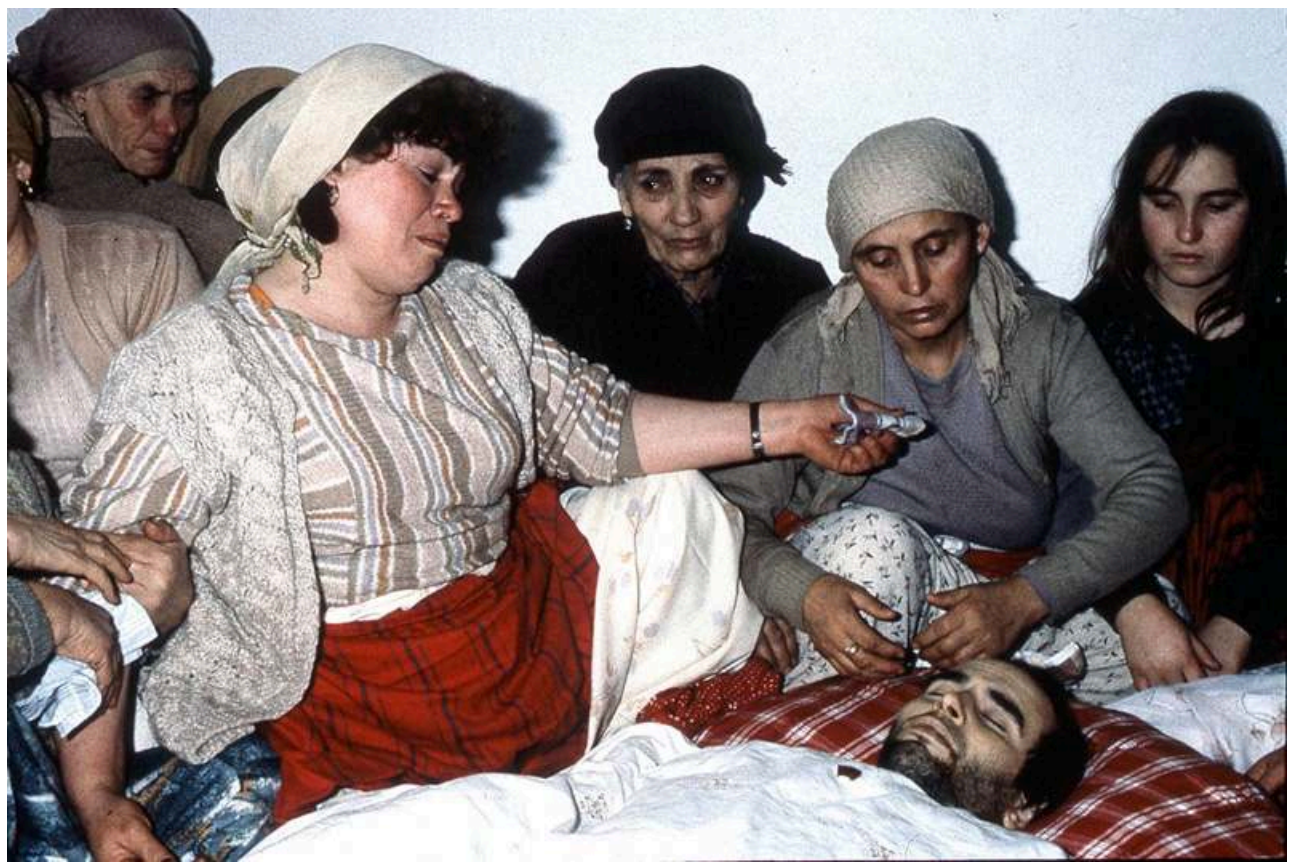

Dusko Despotovic, Veillée funèbre au Kosovo, 1990

Photographie (D.R.) 
13 pour rester dans cette pièce. L'équipe de télévision une fois sortie, l'obscurité relative de la lumière ambiante reprend ses droits. Mérillon se souvient parfaitement de la qualité lumineuse si particulière qui régnait autour du corps : il ne veut rien dire de la Stimmung, de l'atmosphère psychique et pathétique si intense à ce moment d'affliction collective, mais il décrit avec une précision surprenante l'Atmosphäre lumineuse de la pièce. Le froid, dehors, et la masse des femmes serrées les unes contre les autres, dedans, tout cela, en ce deuxième jour de veillée funèbre, avait formé sur la fenêtre (et probablement dans l'air lui-même) une buée qui, dit Mérillon, «adoucissait » toutes choses. C'est dans cette paradoxale douceur de l'air qu'il prendra quelques images de l'inexorable douleur. La pellicule de cent ASA a été poussée à deux cents, le temps de pose est lent - quinzième ou trentième de seconde - l'objectif est un trente-cinq millimètres.

14 L'une de ces images ${ }^{8}$ - dont les cadrages varient, de toute façon, très peu, l'exiguïté de la pièce et la situation elle-même rendant tout mouvement difficile - est une sorte de chef-d'œuvre (fig. 7).

Fig.7

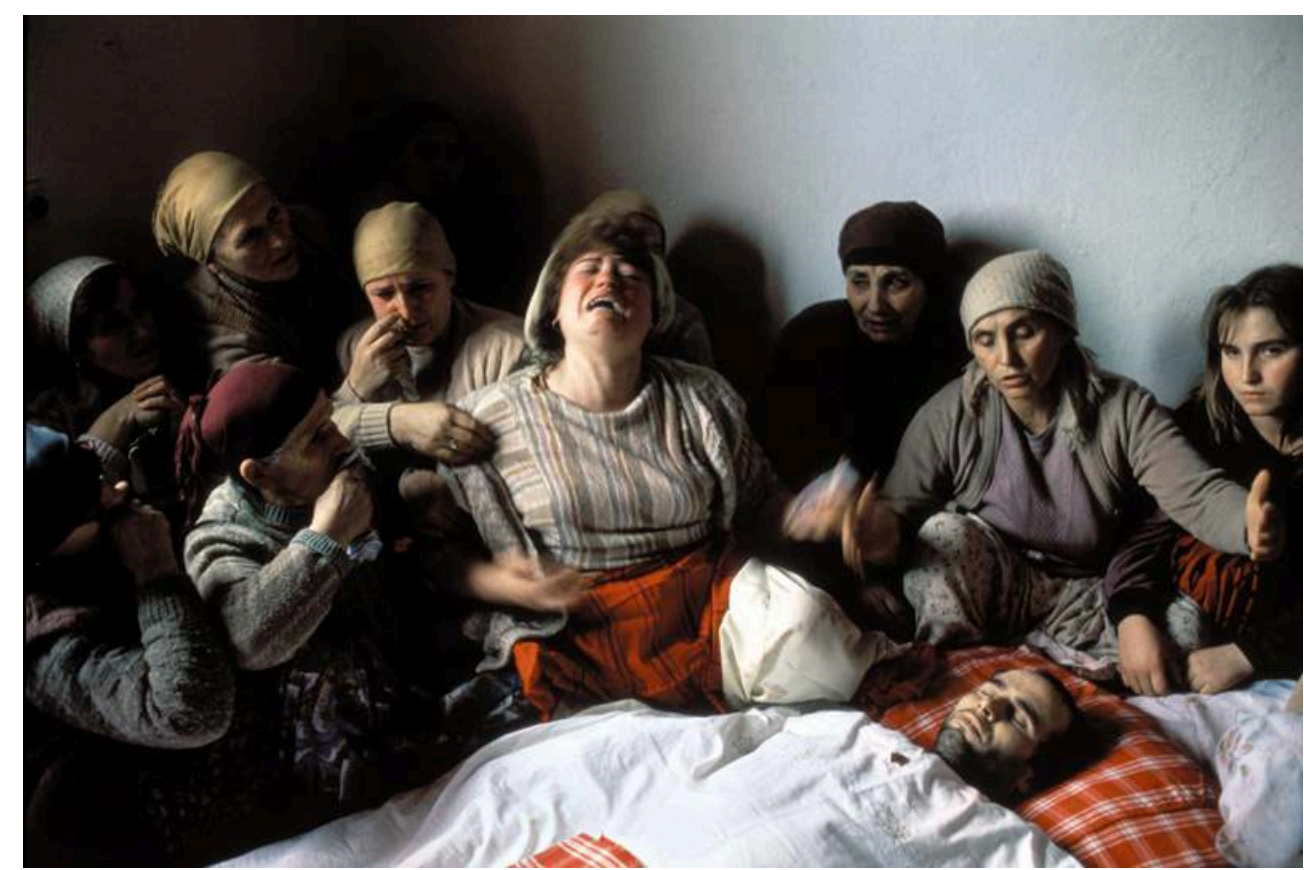

Georges Mérillon, Nagafc, 29 janvier 1990. Veillée funèbre au Kosovo autour du corps de Nasimi Elshani, tué lors d'une manifestation pour l'indépendance du Kosovo

Photographie (Agence Gamma)

Inconnu de son auteur, il va de soi (du moins sur le moment). Il pense alors, simplement, avoir pris en photo ce que l'équipe de télévision a pris en vidéo, les deux médiums étant censés témoigner de la même scène, c'est-à-dire des mêmes corps, du même espace et du même temps. De fait, les diapositives de Mérillon vont suivre, grâce au caméraman Daniel Lévy qui rentre à Paris, le même chemin que les cassettes vidéo destinées au journal télévisé de France-2. Mérillon a juste le temps de griffonner une légende pour sa série photographique : Nagafc, 29 janvier 1990. Veillée funèbre au Kosovo autour du corps de Nasimi Elshani, tué lors d'une manifestation pour l'indépendance du Kosovo.

Images Re-vues, Hors-série 1 | 2008 

photographie de "veillée funèbre ». L'image est publiée pour la première fois au printemps 1990 dans L'Express, puis - en double page, mais dans un contexte qui n'a déjà plus rien à voir avec les événements du Kosovo - dans Le Figaro magazine. Fait curieux, la première personne à avoir pris publiquement cette photographie au sérieux semble avoir été François Mitterand, à qui le magazine VSD demande, fin 1990, de commenter son "Journal de l'année ». En marge de la Veillée funèbre, Mitterand écrit deux phrases dont la première semble insister sur la dimension transhistorique - et, d'abord, esthétique - de l'image, tandis que la seconde évoque sans ambiguïté la leçon historique et politique que l'on peut tirer de cette image :

«Comment ne pas penser à une toile de Mantegna ou de Rembrandt ? La colère et la douleur ont toujours le même visage. L'un des plus graves problèmes qui attend l'Europe de cette fin de siècle est celui des minorités. Il faut s'y attaquer au plus tôt ${ }^{9} . »$

En février de l'année suivante, alors que fait rage la Guerre du Golfe - Georges Mérillon étant sur place et tâchant, par tous les moyens, de contourner la censure établie par l'armée américaine ${ }^{10}-$, le jury du World Press Prize, présidé par Christian Caujolle, se réunit à $A_{\text {msterdam }}{ }^{11}$. Onze mille images dues à mille trois cents photographes de presse sont passées en revue. Au terme de débats passionnés, les jurés se rendent au point de vue qu'il faut primer une image plutôt que l'événement qu'elle documente. Et c'est ainsi que la somptueuse photographie de Mérillon, malgré ce qui apparaît, à l'époque, comme un "petit sujet ${ }^{12}$ " (small subject), est primée "photo de l'année ». Christian Caujolle évoquera cette "image exceptionnelle » sous le double point de vue de sa valeur esthétique - «qui rappelle Rembrandt» - et de sa capacité politique à souligner combien l'événement localisé de Nagafc constitue le symptôme d'un "problème majeur [et] brûlant» pour l'Europe à venir ${ }^{13}$. Ce que l'histoire n'allait malheureusement pas démentir.

Et c'est ainsi que la Veillée funèbre de Georges Mérillon, célébrité aidant, sera devenue la Pietà du Kosovo. Qui donc a substitué au factuel de la légende initiale cette dimension iconographique et culturelle, voire cultuelle? Mérillon m'assure qu'il n'en sait rien. Caujolle, de son côté, a émis toutes les réserves au sujet d'un vocabulaire pictural et religieux qui a aussi requalifié une photographie d'Hocine - sur laquelle Convert travaille également -, photographie intitulée par le journaliste Michel Guerrin La Madone de Benthala ${ }^{14}$ (fig. 8). 
Fig.8

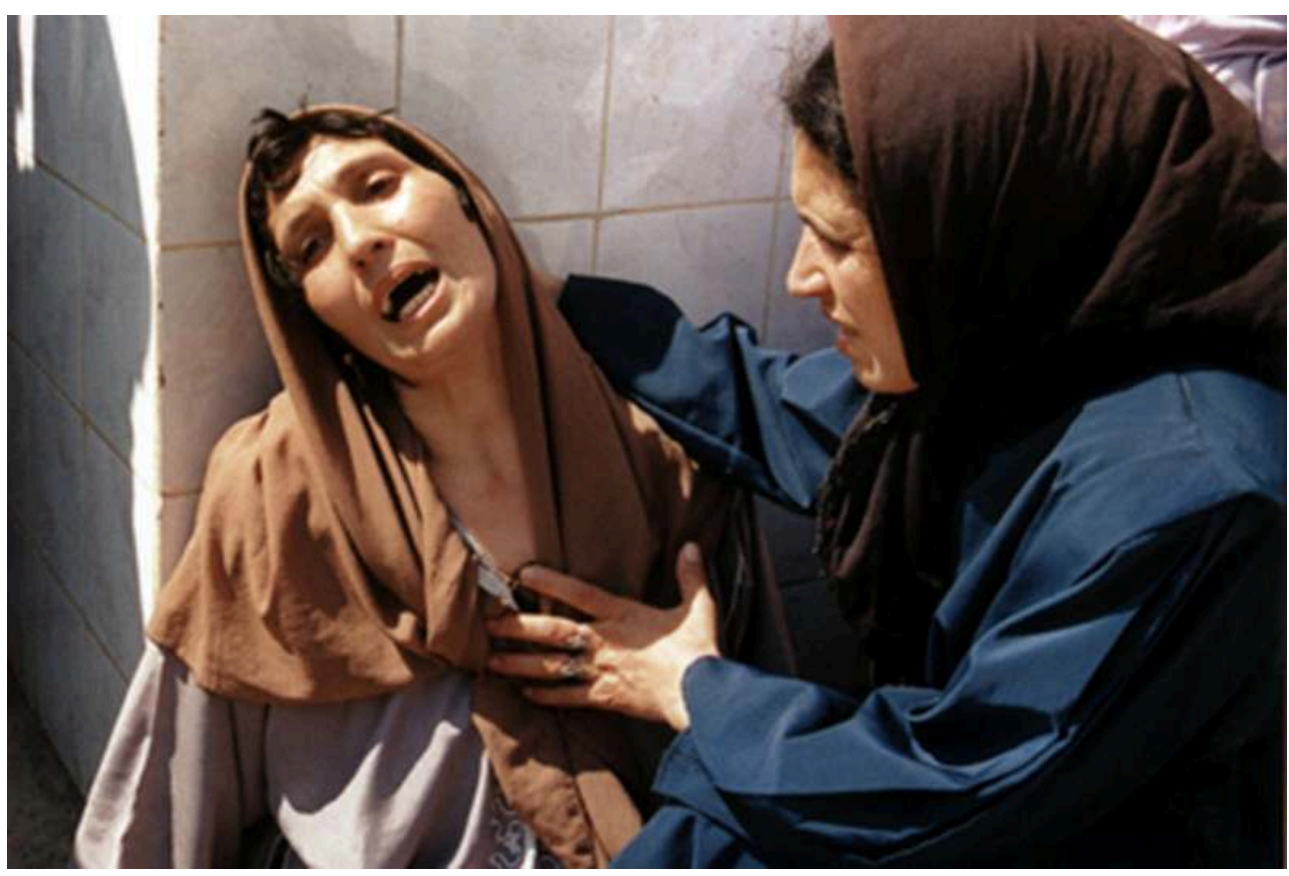

Hocine, Madone de Benthala, 1997

(Agence AFP)

19 Le danger d'un tel vocabulaire, consiste, bien entendu, à oublier l'information dans la compassion. Ne risque-t-on pas de se satisfaire d'un pur affect (pathos, passivité) pour éviter la question politique comme telle (éthos, possibilité de l'action)? Ne veut-on pas, fût-ce inconsciemment, troquer une immédiate empathie contre la patiente compréhension historique que requièrent les événements?

De plus, Pietà et Madone se réfèrent directement à une iconographie chrétienne. Or, les réalités que ces deux photographies documentent - au Kosovo comme en Algérie - se réfèrent, elles, à des tragédies qui adviennent en monde musulman. Ne colonise-t-on pas la douleur des gens de Nagafc ou de Benthala en la plaçant sous une grille sémantique qui a le Christ et la Madone pour modèles ultimes et explicites? On comprend le côté brûlant du débat, à une époque et dans des lieux où christianisme et islam semblent se confronter directement, le Kosovo étant exemplaire à ce titre puisque les Kosovars albanophones, en grande partie musulmans, s'opposent aux Serbes qui, eux, sont généralement chrétiens orthodoxes. Le débat ira, de fil en aiguille, jusqu'à mettre en face de ces photographies publiées dans la presse occidentale le fameux interdit coranique de la représentation - manière un peu rapide, il faut le dire, de disqualifier ces « icônes » par trop pathétiques.

21 Le problème est, évidemment, beaucoup plus complexe que le ne laisse entrevoir cette mise en opposition trop générale. Les premières personnes à tenir l'image de Mérillon pour importante furent les femmes elles-mêmes - les femmes musulmanes - de la famille Elshani : la Veillée funèbre fut accrochée au mur de la chambre de Nasimi avant de circuler dans toute la région. Et, lorsque l'armée serbe, avec les milices paramilitaires des "Tigres d'Arkan", investirent le village de Nagafc le 12 avril 1999 pour le piller et le réduire en cendres, Aferdita, la jeune sœur du mort - on la voit tout à droite de l'image, c'est encore une adolescente en 1990, ses cheveux ne sont pas 
couverts (fig. 7) -, eut tout juste le temps d'enterrer les photographies dans le jardin, comme Mérillon devait l'apprendre en juin 1999, revenant sur les lieux à la recherche des survivants ${ }^{15}$.

Le problème est complexe, parce que les images impliquent une durée qui va bien au-delà du temps qu'elles représentent ou qu'elles documentent. Nous aurons, une fois de plus, à vérifier combien les images fonctionnent selon cette "dialectique » temporelle dont parlait si bien Walter Benjamin, ou selon cet enchevêtrement culturel de «migrations » et de "survivances» dont parlait, de son côté, Aby Warburg ${ }^{16}$. Il faudra donc, pour comprendre de telles images, interroger leur destin - au sens où Freud a pu parler, notamment, d'un "destin » des pulsions, ou bien au sens visé par Georges Bataille lorsqu'il affirmait, dans son ultime conférence du Collège de Sociologie, en 1939: "Mettre en face de la destinée demeure à mes yeux l'essentiel de la connaissance ${ }^{17}$. "

Mais qu'est-ce qu'un destin ? C'est ce que l'histoire produit au-delà d'elle-même : c'est ce qui l'engage vers un passé dont elle ne se souvient plus et vers un futur qu'elle ne connaît pas encore. La photographie de Mérillon, certes, se réfère à un événement spécifique, elle doit donc se penser à partir des remous de l'histoire qu'elle documente fragmentairement (puisqu'elle ne documente qu'une très courte durée de l'événement, son quinzième ou trentième de seconde). La sculpture de Convert, quant à elle, offre à cette image un devenir-œuvre qui lui confère une densité, une matérialité, une monumentalité, un relief supplémentaires - ce relief fût-il en négatif, cette matière fût-elle de cire et cette densité fût-elle trou et décoloration. L'œuvre de Convert et l'image de Mérillon appartiennent sans aucun doute à des lieux différents de la culture, elles n'en existent pas moins selon une époque commune, et c'est cette époque qu'il faudra d'abord interroger à travers les deux régions apparemment fort éloignées que forment les œuvres de l'art et les images de la guerre.

Il sera donc nécessaire - et je ne fais là que redire le credo élémentaire de tout historien qui se respecte - de situer cette image de presse et cette œuvre d'art dans leurs contextes respectifs : l'histoire des conflits balkaniques où les événements de Nagafc prennent leur source, l'histoire du photojournalisme de guerre dont Georges Mérillon est un acteur, l'histoire de l'art contemporain à laquelle appartient l'œuvre de Pascal Convert. Mais cela - le temps de l'histoire - ne nous renseigne que sur un aspect seulement des choses. Il entre justement dans le pouvoir des images de montrer ce que l'histoire produit au-delà d'elle-même. Gilles Deleuze l'a dit à sa - belle - façon : «Il me semble évident que l'image n'est pas au présent. [...] L'image même, c'est un ensemble de rapports de temps dont le présent ne fait que découler, soit comme commun multiple, soit comme plus petit diviseur. Les rapports de temps ne sont jamais vus dans la perception ordinaire, mais ils le sont dans l'image, dès qu'elle est créatrice. Elle rend sensibles, visibles, les rapports de temps irréductibles au présent ${ }^{18}$.

Ces rapports de temps irréductibles au présent - irréductibles, par conséquent, à l'illusion d'une coïncidence parfaite entre le temps de l'image et celui de l'événement, voire de l'époque, d'où elle tire son existence même - n'apparaissent qu'au terme d'une interprétation, c'est-à-dire de ce que Freud a nommé une «construction dans l'analyse ${ }^{19}$ » : construction de sens involuée dans l'analyse de son matériau, et qui n'a elle-même sens qu'à travers une construction de la durée. Il s'agit bien de mettre l'histoire " en face de sa destinée »: de la confronter au passé qui l'engage mais dont elle ne souvient plus, ainsi qu'au futur où elle s'engage mais dont elle ne sait encore rien. Il s'agit de construire l'historicité selon la mémoire et selon le désir qui la portent 
inconsciemment. Or, l'art de cette construction - de cette connaissance - passe par une certaine pensée et par une certaine pratique du montage. C'est ce qu'Aby Warburg a entrepris pour son compte, dans les dernières années de sa vie, au titre d'un atlas d'images qu'il n'a justement pas intitulé Clio, mais bien Mnémosyne ${ }^{20}$.

Une planche de cet atlas, préparée par plusieurs versions antérieures, a justement pour thème l'expression de la douleur extrême à travers l'iconographie - mais aussi, implicitement, à travers le motif théorique développé par Lessing - de Laocoon ${ }^{21}$. Elle doit être comprise selon le projet, esquissé par Warburg dès 1889, de comprendre les représentations occidentales du pathos, depuis la Grèce hellénistique jusqu'à Donatello et au-delà, selon un point de vue anthropologique diamétralement opposé aux canons esthétiques de Winckelmann et de Lessing ${ }^{22}$. Non par hasard, cette planche est directement précédée par l'un des nombreux montages dédiés dans l'atlas à la figure paradigmatique de Ninfa: c'est un ensemble d'images où le motif de la féminité se rapproche dangereusement de la négativité violente et meutrière ${ }^{23}$. La planche qui suit clôt en toute logique cette espèce de dialectique: elle est consacrée aux motifs du cadavre déposé, du deuil et de la lamentation ${ }^{24}$ (fig. 9).

Fig. 9

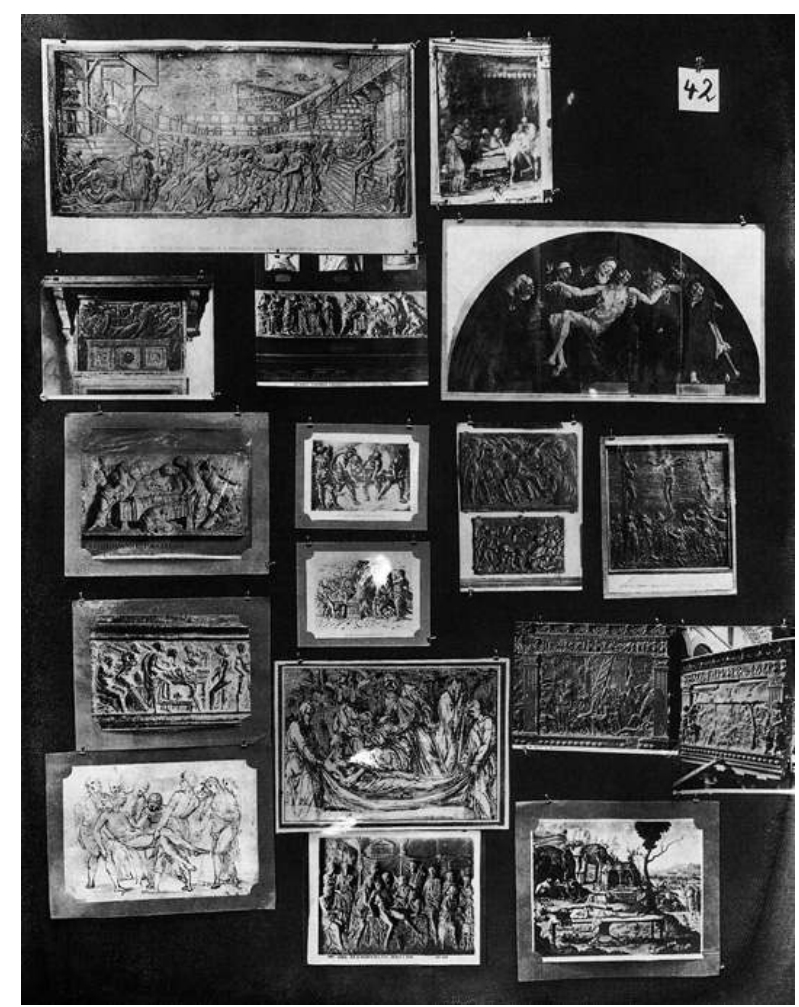

Aby Warburg, Bilderatlas Mnemosyne, 1927-1929. Planche 42 : Pathos de la douleur dans son inversion énergétique (Penthée, Ménade à la croix). Plainte funèbre, héroïsée. Cantiques de lamentation. Mort du Rédempteur. Mise au tombeau. Méditation funèbre. Londres, The Warburg Institute

Des bacchantes païennes aux pleureuses chrétiennes, en passant par la kinah juive ou la plainte musulmane, c'est tout le motif - essentiellement tragique - d'une Ninfa dolorosa que Warburg esquissait dans les planches de son atlas. Mettre la photographie de Georges Mérillon et la sculpture de Pascal Convert "en face de leur destinée ", ce serait donc, en toute logique, commencer par le remontage d'un vaste atlas des lamentations où ces images trouveraient esquissées leurs relations formelles, leurs 
circulations temporelles de longue durée, leurs puissances anthropologiques. Destinée tendue entre antiquité et modernité, avec, sans doute, comme charnière historique, les images cruciales que Donatello inventa au $\mathrm{XV}^{\mathrm{e}}$ siècle pour figurer ce que le pathos et l'expression de la douleur veulent « dire », dans l'excès de toute rhétorique.

Dans son bref et remarquable article sur le cinéma de Guy Debord, Giorgio Agamben a insisté sur le "caractère éminemment historique " de l'image; il reconnaît dans le " projet d'Aby Warburg » les moyens de comprendre ce qui fait de cette histoire autre chose qu'une simple chronologie ${ }^{25}$. Du Bilderatlas warburgien à Godard ou à Debord - il faudrait au moins ajouter Chris Marker à cette filiation, sans compter, pour les années trente, Eisenstein ou le Bataille de Documents -, c'est bien le montage qui constitue le paradigme même de toute construction (qu'elle ait une visée épistémique ou esthétique ne change rien sur ce plan: dans les deux cas, la «construction dans l'analyse » ne va pas sans construction dans la forme). Or, les conditions de possibilité fondamentales du montage - ses "transcendantaux", écrit Agamben - sont reconnues pour être la répétition et l'arrêt.

La répétition, nous le savons mieux depuis Kierkegaard, Nietzsche, Freud ou Gilles Deleuze, « restitue la possibilité de ce qui a été, le rend à nouveau possible. C'est là que réside la proximité entre la répétition et la mémoire. Car la mémoire ne peut pas non plus nous rendre tel quel ce qui a été. Ce serait l'enfer. La mémoire restitue au passé sa possibilité. [Elle] est pour ainsi dire l'organe de modalisation du réel, ce qui peut transformer le réel en possible et le possible en réel ${ }^{26}$. " L'arrêt, quant à lui, s'éclaire autant de l'« interruption révolutionnaire » théorisée par Walter Benjamin que de la " césure » reconnue par Hölderlin en tant que respiration profonde du poème ${ }^{27}$. Mais il ne faut pas dire que la césure " arrête le rythme » en interrompant le déroulement des mots et des représentations ${ }^{28}$. Il faut dire, au contraire, qu'elle constitue comme rythme le rapport ainsi établi entre la répétition et ses arrêts ou différences. Le montage est rythme. En ce sens est-il capable de donner le phrasé de l'histoire dont Jacques Rancière a si bien parlé à propos, justement, du cinéma de Godard ${ }^{29}$. D'un côté il complexifie, voire obscurcit la visibilité des choses $^{30}$; d'un autre il musicalise les choses, c'est-à-dire révèle leurs plus secrètes « dynamographies ${ }^{31}$ ».

"L'image en tant que telle ", écrit pour sa part Giorgio Agamben - suivant en cela un des principes théoriques fondamentaux de La Société du spectacle - n'est que «zone d'indécidabilité entre le vrai et le faux ${ }^{32}$. » Proposition qui va de pair avec une critique radicale des images de presse et de télévision : «Les médias nous donnent toujours le fait, ce qui a été, sans sa possibilité, ils nous donnent donc un fait par rapport auquel on est impuissant. Les médias aiment le citoyen indigné, mais impuissant. C'est même le but du journal télévisé. C'est la mauvaise mémoire, celle qui produit l'homme du ressentiment ${ }^{33}$. » Ce diagnostic n'est juste qu'à un certain degré de généralité. Pascal Convert, qui connaît le fonctionnement intime, technique et idéologique, des images télévisées et partage globalement ce pessimisme politique ${ }^{34}$, a pourtant consacré un temps considérable - donner du temps, quel meilleur hommage ? - à des images de presse capables, selon lui, d'ouvrir notre regard sur leurs propres " possibilités ", donc de produire cette «bonne mémoire » qu'évoque Agamben. Comment cela est-il possible, si ce n'est en refusant toute généralité et en construisant la durée par un montage réglé d'images singulières prises dans le grand rhizome de leurs relations ${ }^{35}$ ? 


\section{NOTES}

1. Ce travail comporte trois sculptures monumentales présentées ensemble dans la collection du Musée d'Art moderne Grand-Duc Jean (Luxembourg). L'œuvre dont nous parlons date de 1999-2000. Elle est en cire, résine et cuivre. Ses dimensions sont de 224 x 278 x $40 \mathrm{~cm}$. L'artiste l'a intitulée : Sans titre (inspirée de Veillée funèbre au Kosovo, photographie de Georges Mérillon, 1990).

2. Euripide, Les Bacchantes, trad. J. et M. Bollack, Paris, Minuit, 2005, p. 27.

3. C. Greenberg, "Towards a Newer Laocoon» (1940), The Collected Essays and Criticism, I. Perceptions and Judgments, 1939-1944, éd. J. O’Brian, Chicago-Londres, The University of Chicago Press, 1986, p. 23-38.

4. G. E. Lessing, Laocoon (1766), trad. A. Courtin (1866) revue par J. Bialostocka, Paris, Hermann, 1990, p. 45.

5. Ibid., p. 45-46 et 55-56. Sur le contexte philosophique de ce débat, cf. S. Richter, Laocoon's Body and the Aesthetics of Pain: Winckelmann, Lessing, Herder, Moritz, Goethe, Detroit, Wayne State University Press, 1992.

6. P. Convert, Entretien avec Georges Mérillon, 2003 (document vidéographique).

7. Ibid.

8. Georges Mérillon se souvient avoir réalisé une pellicule entière. Mais il ne lui reste aujourd'hui que cinq autres images de la scène : quatre d'entre elles sont prises d'un angle un peu biaisé, la dernière est cadrée verticalement. Quant aux rushes de Véronique Taveau, ils sont, en toute logique télévisuelle, définitivement perdus.

9. F. Mitterand, « 23 [sic] janvier 1990. Le Kosovo pleure ses morts », VSD, n 694, 20-26 décembre 1990 (« Numéro collection. 1990 : Le journal de l'année par François Mitterand »), p. 40-41.

10. Les trois principales agences photographiques françaises - Sygma, Gamma et Sipa - ayant éta écartées du pool américain, quelques photographes audacieux, dont Mérillon, se sont associés sous le label humoristique FTP (pour Fuck The Pool) et ont tenté, à leurs risques et périls, de tirer des images non officielles de cette guerre très contrôlée. Cf. M. Guerrin, « Les francs-tireurs de la photo face à la censure ", Le Monde, 20 février 1991, p. 5.

11. Jury composé de dix membres : Christian Caujolle (président), Zevi Ghivelder (Brésil), David Goldblatt (Afrique du Sud), Vincent Mentzel (Pays-Bas), Randy Miller (USA), Daniela Mrazkova (Tchécoslovaquie), Kasumiko Murakami (Japon), Raghu Rai (Inde), Ruud Taal (secrétaire) et Vladimir Vyatkin (URSS).

12. G. Mérillon, «I Like to Capture Strong Emotions », World Press Photo Newsletter, juillet 1991, p. 2.

13. C. Caujolle, "The Paradox of Contemporary Press Photography », ibid., p. 4. Id., "Foreword", World Press Photo 1991, La Haye-Londres, World Press Photo Holland Foundation-Thames and Hudson, 1991, p. 5.

14. P. Convert, Entretien avec Christian Caujolle, 2003 (document vidéographique). Cf. M. Guerrin, «Une Madone en enfer », Le Monde, 26 septembre 1997, p. 12.

15. G. Mérillon, Texte inédit (1999) utilisé partiellement dans «Tout le martyre kosovar dans l'histoire d'une famille ", Paris Match, n² 2615, 8 juillet 1999, p. 24-29. Cf. également A. Cojean, « Retour sur images : le martyr du Kosovo », Le Monde, 28 août 1997, p. 11.

16. Cf. G. Didi-Huberman, Devant le temps. Histoire de l'art et anachronisme des images, Paris, Minuit, 2000, p. 85-155. Id., L'Image survivante. Histoire de l'art et temps des fantômes selon Aby Warburg, Paris, Minuit, 2002.

17. G. Bataille, «Collège de sociologie » (1939), CEuvres complètes, II, Paris, Gallimard, 1970, p. 365.

18. G. Deleuze, «Le cerveau, c'est l'écran» (1986), Deux Régimes de fous. Textes et entretiens, 1975-1995, éd. D. Lapoujade, Paris, Minuit, 2003, p. 270. 
19. S. Freud, "Constructions dans l'analyse » (1937), trad. collective dirigée par J. Laplanche, Résultats, idées, problèmes, II. 1921-1938, Paris, PUF, 1985,p. 269-281.

20. A. Warburg, Gesammelte Schriften, II-1. Der Bilderatlas Mnemosyne, éd. M. Warnke et C. Brink, Berlin, Akademie Verlag, 2003 ( $2^{\mathrm{e}}$ édition corrigée).

21. Ibid., p. 74-75, planche 41a : «Pathos de la douleur. Mort du prêtre » (Leidenpathos. Tod des Priesters).

22. Id., Entwurf zu einer Kritik des Laokoons an Hand der Kunst des Quattrocento in Florenz (« Esquisse d'une critique du Laocoon à la lumière de l'art florentin du Quattrocento ») (1889), Londres, Warburg Institute Archive, III, 33.2.4.

23. Id., Gesammelte Schriften, II-1. Der Bilderatlas Mnemosyne, op. cit., p. 72-73, planche 41 : «Pathos de l'anéantissement. Sacrifice. La nymphe comme sorcière. Libération du pathos » (Vernichtungspathos. Opfer. Nympha als Hexe. Freiwerden des Pathos).

24. Ibid., p. 76-77, planche 42 : «Pathos de la douleur dans son inversion énergétique (Penthée, Ménade à la croix). Plainte funèbre, héroïsée. Cantiques de lamentation. Mort du Rédempteur. Mise au tombeau. Méditation funèbre (Leidenpathos in energetischer Inversion [Pentheus, Mänade am Kreuz]. Bürgerliche Totenklage, heroisiert. Kirchl. Totenklage. Tod des Erlösers. Grablegung. Todesmeditation).

25. G. Agamben, « Le cinéma de Guy Debord » (1995), Image et mémoire. Écrits sur l'image, la danse et le cinéma, Paris, Desclée de Brouwer, 2004, p. 88-89.

26. Ibid., p. 91

27. Ibid., p. 92-93.

28. Ibid., p. 93.

29. J. Rancière, «La phrase, l'image, l'histoire » (2002), Le Destin des images, Paris, La Fabrique, 2003, p. 41-78.

30. Cf. P. A. Sitney, Modernist Montage : The Obscurity of Vision in Cinema and Literature, New York, Columbia University Press, 1990.

31. Cf. G. Didi-Huberman, L'Image survivante, op. cit., p. 169-190 et 452-505.

32. G. Agamben, «Le cinéma de Guy Debord », art.cit., p. 95.

33. Ibid., p. 91-92.

34. Cf. P. Convert, «Des images en mercure liquide», Art Press, $n^{\circ} 251,1999$, p. 39-49. Id., « Des images figées », ibid., $\mathrm{n}^{\circ}$ hors-série, 2001 («Représenter l'horreur »), p. 40-47. Id., « Une distance aux choses. Entretien avec Raymond Depardon», ibid., n²73, 2001, p. 29-35. Id., "Images passages », ibid., n spécial 25, 2004 ("Images et religions du livre »), p. 90-95.

35. Ces pages sont extraites d'un travail de longue haleine sur les Pathosformeln de la lamentation.

\section{INDEX}

Mots-clés : débat esthétique, dimension iconographique, durée, expérience visuelle, image visuelle, mémoire, perception, repère de signification, temporalité 


\section{AUTEUR}

\section{GEORGES DIDI-HUBERMAN}

Né en 1953. Philosophe et historien de l'art, enseigne à l'EHESS (Paris). Il a séjourné à Rome

(Académie de France), à Florence (Villa I Tatti-Harvard University Center for Italian Renaissance Studies), à Londres (Institute of Advanced Study, Warburg Institute). Il a enseigné dans plusieurs universités étrangères (Johns Hopkins, Northwestern, Berkeley, Courtauld Institute, Berlin). Il a reçu le Hans-Reimer-Preis de la Aby-Warburg-Stiftung (Hambourg) et le prix Humboldt (2006). Il a dirigé plusieurs expositions, dont L'Empreinte au Centre Georges Pompidou (Paris,1997) et Fables du lieu au Studio national des Arts contemporains (Tourcoing, 2001). Il a publié une trentaine d'ouvrages sur l'histoire et la théorie des images, notamment, pour les plus récents : Ouvrir Vénus. Nudité, rêve, cruauté(Gallimard, 1999) ; Devant le temps. Histoire de l'art et anachronisme des images (Minuit, 2000) ; L'Image survivante. Histoire de l'art et temps des fantômes selon Aby Warburg (Minuit, 2002) ; Ninfa Moderna. Essai sur le drapé tombé (Gallimard, 2002) ; Images malgré tout (Minuit, 2003) ; Mouvements de l'air : Étienne-Jules Marey, photographe des fluides (Gallimard, 2004). Gestes d'air et de pierre. Corps, parole, souffle, image (Minuit, 2005). Le Danseur des solitudes (Minuit, 2006). Ex voto. Image, organe, temps (Bayard, 2006). L'image ouverte. Motifs de l'incarnation dans les arts visuels(Gallimard, 2007). 